\title{
A Summary of the Analysis of Hindemith's Works
}

\author{
Cui Jia \\ Music and Dance Academy at Zaozhuang College, Shandong, China, 277160
}

Keywords: Hindemith; composing theory; practical application

\begin{abstract}
As a famous composer of the 20th century, Hindemith is also a music theorist and educator. His music theory is very comprehensive, rigorous and wide-ranging. In his theory of music, he elaborated the application of various music techniques in detail and made a significant contribution to the development of music art. In the "Composing Techniques" by Hindemith, the theoretical system of composing techniques he created has modernity and practicality and also plays an important role in the field of studying modern music. This paper will conduct a more in-depth analysis of Hindemith's theory of composition and publish a little insight into the practical application of the theory.
\end{abstract}

\section{Introduction}

Paul Xing Demit is a versatile musical genius in Germany. His love of music has involved him in music composition, performance, theory, teaching, command and other fields. His masterpiece has a symphony, "The painter Matisse." ", orchestral "Orchestra Concerto" and so on. His creations cover almost all kinds of musical genres and his works, teaching and music have a profound impact on modern composers.

In the early 20th century, neo-classical tendencies were expressed in the music creation of Xing Demit and this tendency became stronger and stronger. He tried to combine the musical characteristics of the Bach period with the musical style of the time and opposed the exaggerated and impulsive feelings of late romanticism. In his works such as "Piano Music", he has systematically studied and developed a polyphonic technique that has never been seen before, forming a new Baroque style and finding a new but logical set. Counter method. In Xing Demit's view, tonality is actually like gravity. It is a kind of force in nature. Therefore, even if it seems to be uncoordinated in his music composition, it will always end in harmony and simplicity.

In his "Composing Techniques", Xing Demit elaborated a new set of theory of composing theory through his exploration and practice. As a music theorist and music educator, Xing Demit believes that educating students is to cultivate the future composer, so he regards educating students as a very important task. It is because of in the theory of composition of Xing Demit practicality is the most representative feature. The system of composing theory he summed up is not only comprehensive, but also very rigorous, not only closely related to artistic expression, but also has considerable Scientific basis, it has become one of the most important theories in modern music. This paper will start with the analysis of its specific application from the harmony theory system in Xing Demit's theory system of composition ${ }^{[1]}$. 


\section{Xing Demit's harmony theory analysis}

This is the most valuable part of Xing Demit's theory of composition. In this system of harmony theory, Xing Demit negated the basic principles of chord formation in traditional harmony theory and formed a new set. The sound system has a wide range of practicality and distinctive modern features.

\subsection{The new chord composition principle in the harmony theory system}

In the traditional harmony theory, the basic principle of chord formation is three-degree superposition, but in Xingdemite's harmony theory system, the composition of chords is composed of sequence 1 and interval and according to the interval Nature and value, the chord can be divided into two parts and the classification is based on whether there is a three-tone interval in the chord. In Xing Demitt's view, the most prominent feature of the three-tone is the uncertainty and if the chord contains the three-tone, then the chord has some uncertainty. So according to this characteristic, Xingdemute divides the chord into a class A chord and a class B chord. The class A chord is a chord that does not contain a tritone and the class B chord is a chord that contains a tritone. This approach breaks through the traditional pattern of chords with equidistant intervals, providing a broader space for composers to create originality in their creations. After the composition of the chord was redefined by Xing Demit, the chord root also appeared in a new meaning. Since the chord is composed of intervals and each interval contains a root, then in a chord, there must be several different intervals and after finding the best interval by the value of the sequence 1 , The root of this best interval is the root of the chord.

\subsection{Harmony development logic}

Music can't be composed of only one chord. It must be through the connection of chords and chords to produce the effect of harmony. This kind of music has meaning. The development logic of harmony is shown in the figure 1 below.

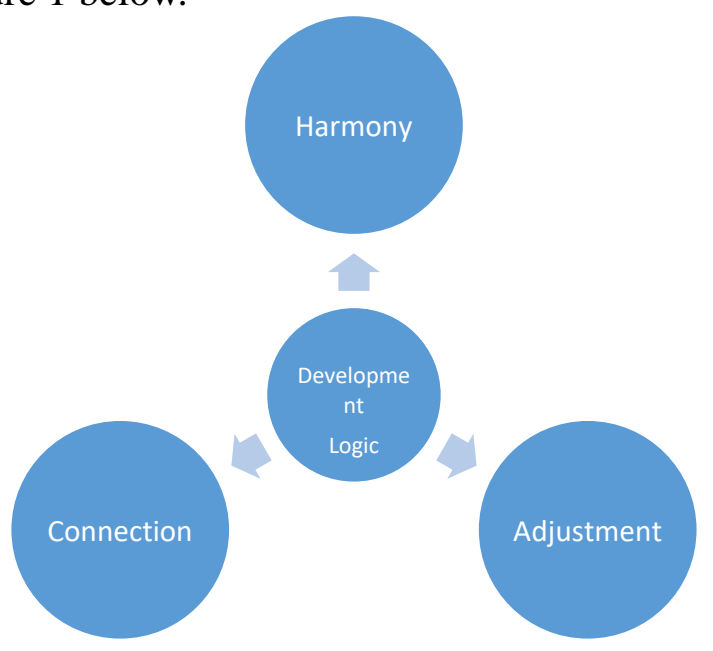

Figure 1 Development logic of harmony

(1) Harmony. If chords contains different degrees of tension are connected together, the tension will rise or fall, resulting in harmonic fluctuations. Therefore, harmonies can only form when chords of different values are combined and this ups and downs may be sudden or gradual. Sudden ups and downs are due to the fact that the chords with large differences in value are connected together, such as from I1-V, or from IIa-IV1, such a harmony can produce sudden fluctuations while 
proceeding; progressive undulations are the same Composed of a chord in a chord group. Harmony and undulations, two skeletons and part of the voice are all part of the development of harmony. The design of these three parts can be contradictory or consistent and when dealing with the relationship between the three parts. It can reflect the logical laws of harmony development ${ }^{[2]}$.

(2) Chord connection skills. In Hindmit's harmony theory, chords are not random connections, but the intervals formed by the roots of two connected chords are used to measure the value of the two chord connections, thus connecting them as chords. Judgment basis. However, the basis for judging or evaluating based on this basis is not comprehensive. Therefore, Xing Demit will compensate each other for different forces in the harmony movement as another evaluation criterion, thus forming another original theoretical principle.

(3) Adjustment. If a class B chord is added to the chord connection and the class B chord contains a three-tone, the resulting tension must have a resistance to resolve the tension. This is only the class A chord. Produce such resistance. In the process of solving the three-tones, it is necessary to apply a new principle in the theory of Xingdemit's harmony, that is, the guiding principle. The guiding sound refers to the best relationship with the root in the three-tones of the B-chord. The sound of that (measured by the sequence 1). However, it is worth noting that the root and tone of the chord are not necessarily the same part. These two principles are only used as a criterion for judging and measuring the value of chord connection and are the theoretical basis for chord selection ${ }^{[3]}$.

\section{Specific application of harmony theory}

\subsection{Harmonic design}

In the process of creating musical works, no matter what period or style of music should take into account the changes in harmonic tension, but in the traditional theory, this harmonic design is not systematically discussed. But Xing Demit did it. In his theory of harmony, the chords are divided into two categories, A and B and there are groups in each class, which gives us a huge chord database. Because the value of the chord connection is different, so how to make the harmony of the harmony can be relaxed and orderly, so that the connection of the chord can reflect the purpose of creation, which is the core of the undulation in design. problem. In the undulating design, the overall design and partial design are included. From the overall design point of view, according to the content of the musical works, the changes of emotions, the overall and partial climax, etc., the overall trend of the harmonic tension of the musical works can be planned and the best parts of each part can be determined. Chord type. From the point of view of the partial design, the two types of skeletons and the overall arrangement can be combined to determine the type of local undulations and the chord group. Before the creation of a musical composition, the style, mood and length of the work should be roughly planned. The key point is to determine how the tension in the ongoing harmony should develop. Is it necessary to relax after tension or relax? What position is in place to determine the chords in each part ${ }^{[4]}$.

\subsection{Designing local harmonic fluctuations}

(1) The harmonies of the presentation part of the work. The materials in this part are generally and the tonality and sound are relatively clear and they are more uniform and regular in the structural unit. Therefore, the "undulation" of the harmony will not be too large and the tension of the chord will not be too high. The three harmonies are shown in Figure 2. 


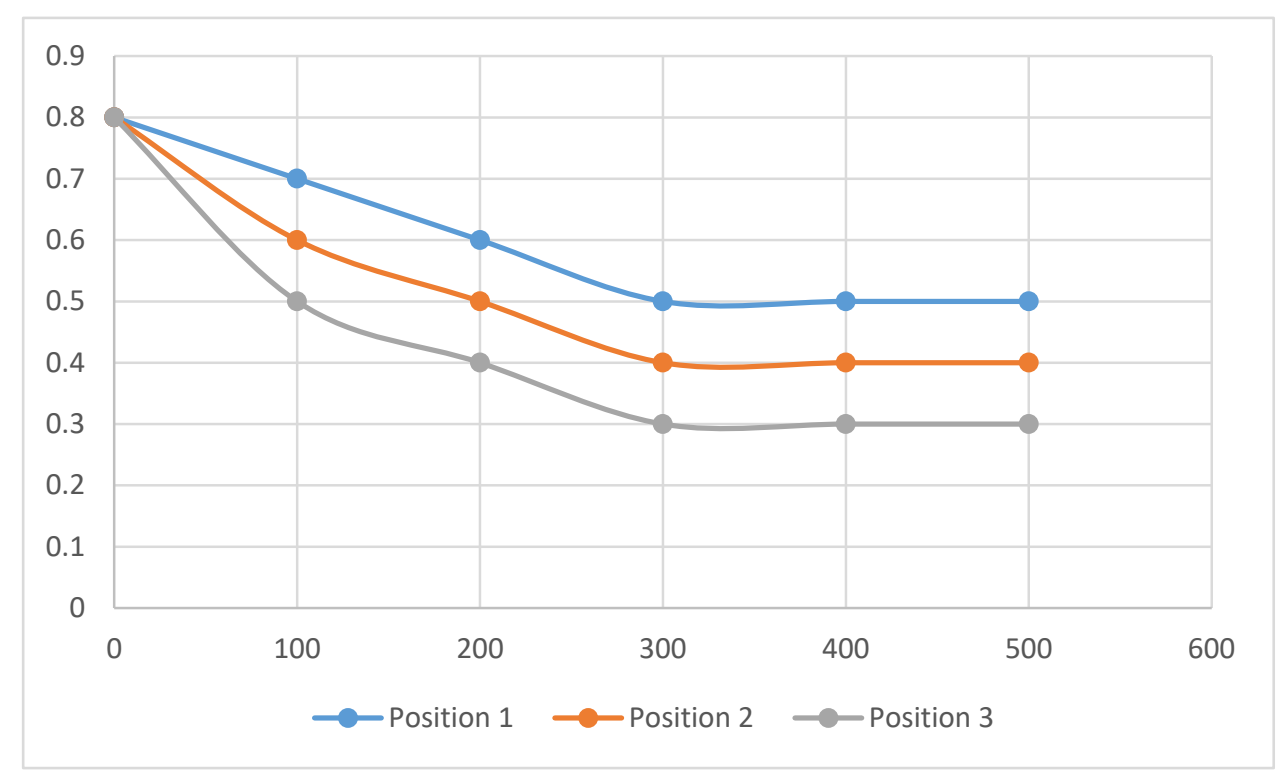

Figure 2 Law of three harmonies

(2) The harmony of the unfolding part of the work. The materials in this part are generally fragmented and fragmented and the tension of the harmony is increased compared with the previous part, until it falls back after entering the overall climax and the structure is not regular and symmetrical, showing a rise - The structural characteristics of the suppression or suppression - Yang or suppression - Yang - suppression, the various structures are interlocking, but in general, before the arrival of the climax, the overall trend of "gradual strength" is reflected until The tension of the chords at the climax points is highest.

(3) The undulating undulations at the end of the work. The type and presentation of the undulations at the end are essentially the same, but the end is mostly a collapsed structure, while the presentation is also an open structure. Therefore, at the end of the work, a stable chord design with high harmonies is usually performed and the undulating trend in this part is fading. But this is only a general situation and special treatment can be done according to the actual situation of the work ${ }^{[5]}$.

\section{Conclusion}

In summary, in the "Composing Techniques" by Xing Demit, the theoretical system of composing techniques he created has modernity and practicality and also plays an important role in the field of studying modern music. In his theory of composing theory, harmony theory is a logically complete and complete part. The concepts and principles of each theory are closely related and closely related and through his harmonic theory, it can analyze any period. The work of any style of composer, the extensive use of this theory is a very prominent feature and when using this theory to create, you can create a different style of work according to the composer's own choice. Therefore, the practicality and modernity of Xing Demit's harmony theory has a very important position in the research field of modern music, which deserves our deeper research and reflection.

\section{References}

[1] Saurabh P. Jangam,Satish Kumar,Shruti Maheshwari. Literature review on analysis of various components of IC engine [J]. Materials Today: Proceedings, 2018,5(9).

[2] Myxuan Huynh,Loredana Gabriela Marcu,Eileen Giles,Michala Short,Donna Matthews,Eva Bezak. Current status of proton therapy outcome for paediatric cancers of the central nervous system - Analysis of the published literature [J]. Cancer Treatment Reviews, 2018,70. 
[3] Xiao Ji,Deepak K. Rajpal,Johannes M. Freudenberg. The essentiality of drug targets: an analysis of current literature and genomic databases [J]. Drug Discovery Today, 2018.

[4] Gene Klein. The effectiveness of peer assessment and a proposal for its analysis using game theory[J]. Journal of Education for Business, 2018, 93(8).

[5] Eugénia Pedro,João Leitão,Helena Alves. Back to the future of intellectual capital research: a systematic literature review [J]. Management Decision, 2018, 56(11). 